University of Wollongong

Research Online

Faculty of Engineering and Information

Faculty of Engineering and Information

Sciences - Papers: Part B

Sciences

2017

\title{
Modelling of the human shoulder girdle as a 6-4 parallel mechanism with a moving scapulothoracic joint
}

\author{
Aibek Niyetkaliyev \\ University of Wollongong, asn936@uowmail.edu.au \\ Shahid Hussain \\ University of Wollongong, shussain@uow.edu.au \\ Prashant K. Jamwal \\ Nazarbayev University, prashant.jamwal@nu.edu.kz \\ Gursel Alici \\ University of Wollongong, gursel@uow.edu.au
}

Follow this and additional works at: https://ro.uow.edu.au/eispapers1

Part of the Engineering Commons, and the Science and Technology Studies Commons 


\title{
Modelling of the human shoulder girdle as a 6-4 parallel mechanism with a moving scapulothoracic joint
}

\author{
Abstract \\ Human shoulder movements involve motions at four different articulations, one of which is the contact \\ between the scapula bone and the ribcage. The shoulder biomechanical models become less reliable \\ when the scapulothoracic (ST) contact, which is not a joint in the anatomical sense, is not considered. On \\ the other hand, constraints posed by the ST contact reduce the number of degrees of freedom (DOF) and \\ introduce the interdependencies between the joint coordinates which in turn complicates the motion \\ planning. However, a minimal parameterization that incorporates the constraints, notably simplifies costly \\ computational procedure related to the model predictions. In this paper, the complex kinematics of the \\ human shoulder is analyzed considering the point-contact model between scapula bone and thorax. Later, \\ replacing the contact constraint with an equivalent kinematic chain and adding parallel kinematic links, \\ the human shoulder girdle is modelled as a 6-4 parallel mechanism. A novel minimal set of independent \\ parameters equal to the number of degrees of freedom is then devised in terms of the parallel \\ mechanism's link lengths and the shoulder joint angles. The proposed parallel mechanism can also \\ emulate the moving ST contact point during the shoulder motions. Finally, the shoulder motion planning \\ method in terms of the time-dependent minimal coordinates is presented.

\section{Disciplines} \\ Engineering | Science and Technology Studies

\section{Publication Details} \\ Niyetkaliyev, A. S., Hussain, S., Jamwal, P. K. \& Alici, G. (2017). Modelling of the human shoulder girdle as \\ a 6-4 parallel mechanism with a moving scapulothoracic joint. Mechanism and Machine Theory, 118 \\ 219-230.
}




\title{
Modelling of the Human Shoulder Girdle as a 6-4 Parallel Mechanism with a Moving Scapulothoracic Joint
}

\author{
Aibek S. Niyetkaliyev ${ }^{\mathrm{a},{ }^{*}}$, Shahid Hussain ${ }^{\mathrm{a}}$, Prashant K. Jamwal ${ }^{\mathrm{b}}$ and Gursel Alici ${ }^{\mathrm{a}}$

 \\ ${ }^{\mathrm{b}}$ Department of Electrical and Electronic Engineering, Nazarbayev University, Astana, Kazakhstan
}

\begin{abstract}
Human shoulder movements involve motions at four different articulations, one of which is the contact between the scapula bone and the ribcage. The shoulder biomechanical models become less reliable when the scapulothoracic (ST) contact, which is not a joint in the anatomical sense, is not considered. On the other hand, constraints posed by the ST contact reduce the number of degrees of freedom (DOF) and introduce the interdependencies between the joint coordinates which in turn complicates the motion planning. However, a minimal parameterization that incorporates the constraints, notably simplifies costly computational procedure related to the model predictions. In this paper, the complex kinematics of the human shoulder is analyzed considering the point-contact model between scapula bone and thorax. Later, replacing the contact constraint with an equivalent kinematic chain and adding parallel kinematic links, the human shoulder girdle is modelled as a 6-4 parallel mechanism. A novel minimal set of independent parameters equal to the number of degrees of freedom is then devised in terms of the parallel mechanism's link lengths and the shoulder joint angles. The proposed parallel mechanism can also emulate the moving ST contact point during the shoulder motions. Finally, the shoulder motion planning method in terms of the time-dependent minimal coordinates is presented.
\end{abstract}

Keywords: shoulder, scapula, joints, kinematics, parallel mechanism, biomechanics.

\section{Introduction}

The shoulder complex is the most mobile part of the human body that provides a broad range of motions for the upper arm. Since it plays a vital role in the human activities of daily living (ADL), its proper functionality is important and as such, there is a need for an appropriate biomechanical model of the shoulder that can simulate complicated upper limb movement patterns. To date, only a few musculoskeletal models for the shoulder and upper limb have been developed that are most commonly used for a variety of purposes [1]: Swedish Shoulder Model [2, 3], Delft shoulder and elbow model (DSEM) [4, 5], Newcastle shoulder model (NSM) [6], Holzbaur's upper extremity model (HM) [7], Anybody model [8], Garner's model [9] and Dickerson's model [10]. Owing to the increasing interest of researchers in kinematic properties of the human shoulder, a number of different shoulder models based on open-loop [11-15] and closed-loop kinematic chains [14, 16-21] have been developed. Characteristically, the open-loop chain shoulder models have rather basic structures which simplify the kinematic and dynamic analyses. However, most of them do not consider the gliding motion of the scapula over the thorax. On the contrary, the closed-loop chain models with higher precision and load carrying capacity can have singular points within their limited workspace. Despite the considerable amount of research that has been done on this topic, the complete analysis of the intricate kinematics of the human shoulder remains a challenging task.

The human shoulder can be considered as a hybrid mechanism whereby the shoulder girdle (thorax, clavicle and scapula) is a positioning and orienting parallel mechanism for the humerus which is serially connected through the glenohumeral $(\mathrm{GH})$ joint. Generally, the sternoclavicular (SC) joint that connects the clavicle to the thorax, the acromioclavicular (AC) joint that connects the scapula to the clavicle and the GH joint are modelled as ideal three degrees of freedom (3-DOFs) ball-and-socket joints. However, a sphere-on-sphere model [22] and deformable joint [23] have also been proposed to model the GH joint.

The scapulothoracic (ST) articulation makes the shoulder girdle a closed kinematic chain constraining the scapula to move over the thorax and reduces the overall DOFs of the shoulder.

\footnotetext{
*Corresponding author at: Northfields Ave, University of Wollongong, NSW, Australia. Tel.: +61421038349

E-mail address: asn936@uowmail.edu.au
} 
Therefore, the gliding motion of the scapula bone is usually modelled using geometrical constraints: a contact between one [20,22], two or three $[5,19,24,25]$ fixed points belonging to the scapula with an ellipsoid (or cone [17]) representing the thorax. In fact, physiologically, this contact point is not fixed on the scapula bone during the shoulder movements [26]. Therefore, the shoulder models with fixed ST contact points may lead to nonphysical scapula movements [20]. The models that have a tangential ST constraint $[27,28]$ (the scapula plane to be normal to the ellipsoid) result in a more physiological ST model [29]. In contrast to rigidly constrained scapula models, the integrated kinematic interaction between the scapula and the humerus, usually referred as scapulohumeral rhythm or shoulder rhythm, leads to another approach with regression equations where the scapula and clavicle joint angles have been defined as a function of the humeral angles [3, 30-33]. However, the drawbacks of these regression models are that they do not respect the ST constraints, cannot describe the independent scapula and humerus motions and cannot distinguish pathological shoulder. In [22], it has been argued that the introduction of the kinematic constraints is more pertinent than the use of couplings between the shoulder joints' coordinates. Further, in order to obtain an adequately modelled shoulder kinematics, the contact constraints have been added to the shoulder rhythm model in [34]. A recently developed OpenSim biomechanical model of the scapulothoracic joint, based on an internal coordinate joint formulation (ellipsoid mobilizer), enforces the motion of the scapula without kinematic surface constraints and describes the scapular kinematics with 4-DOFs [28].

In [19], the human shoulder girdle structure has been modelled as a 2-3 (number of base-top joints) parallel mechanism with the thorax as a base and scapula as a moving platform where the scapulathorax two holonomic constraints have been replaced with two UPS (universal-prismatic-spherical) kinematic chains with passive prismatic pairs. In comparison to the joint angle-description of shoulder kinematics, by modelling the shoulder girdle as a parallel mechanism, a set of independent parameters equal to the number of DOFs were introduced and referred as minimal coordinates. The minimal coordinates that have the advantage of being independent incorporate the constraints. The use of such minimal coordinates considerably facilitates the kinematic motion planning procedure given their independence. A kinematic analysis of the parallel model in [19] led to the construction of three alternative forward kinematic maps and three minimal sets of independent coordinates. However, constructing a dynamic model using these coordinates is somewhat problematic as these coordinate sets need to be mapped back to the joint angle parameterisation and they do not have an immediate physiological implication.

In the light of the above, this paper proposes a model of the human shoulder with the modified parallel shoulder girdle mechanism along with a novel minimal parameterisation of the shoulder model's kinematics. First, the scapulothoracic (ST) articulation is modelled with only one scapula point constrained to move on the surface of the thorax (ellipsoid) which leads to 8-DOFs for the shoulder complex: nine kinematic coordinates subject to one constraint. Then, this contact constraint is replaced with the equivalent UPS kinematic chain with passive prismatic joint which leads to only one additional forward kinematic map. Finally, by introducing four additional UPS links with active prismatic joints, which do not alter the number of DOFs, the human shoulder girdle is modelled as a 6-4 parallel mechanism. The configuration of the 4-DOFs scapula is then parameterised in terms of four active link lengths. This has resulted in a set of minimal independent parameters that can all have a direct geometrical significance and can be easily used in the dynamic analysis of the human shoulder. Subsequently, the forward kinematic modelling of the proposed parallel mechanism is derived in a way such that the ST contact point can move on the scapula plane during the given shoulder motion. In addition, the proposed model can also be adapted for pathological shoulder cases. Finally, using the proposed kinematic model, a feasible parallel mechanism can be designed to have equivalent kinematic properties to those of a human shoulder girdle.

The paper is organized as follows. Section 2 describes the kinematic model of the human shoulder with one ST contact constraint based on the other models from the literature. Section 3 presents the parallel mechanism based model of the human shoulder girdle with its detailed kinematic analysis. In Section 4, a case study with the proposed shoulder parallel mechanism is presented where the minimal coordinates have been used to plan the abduction of the arm in the scapular plane. Finally, Section 5 presents a discussion on the results and limitations of the proposed model. The elaboration on future work concludes the paper. 


\section{A kinematic model of the human shoulder.}

The geometric model of the shoulder and the bony landmarks used in this work are based on recommendations of the International Society of Biomechanics (ISB) [35]. The kinematic model parameterizes the movement of each of the three bones in the shoulder relative to the thorax which is fixed. Each bone is represented by the following bony landmarks shown in Fig. 1: thorax (IJ, PX, T8, C7), clavicle (SC, AC), scapula (AA, TS, AI) and humerus (GH, HU).



Fig.1. The shoulder bony landmarks and coordinate systems ( 0 - thorax, 1 - clavicle, 2 - scapula, 3 humerus). Images are created using OpenSim model [36].

The local coordinate systems (clavicular, scapular and humeral) are centered at the joints around which the corresponding shoulder bones rotate: the clavicle around the SC joint, the scapula around the $\mathrm{AC}$ joint and the humerus around the $\mathrm{GH}$ joint. These reference systems are constructed following the guidelines set by ISB. A subindex is attributed to each reference frame. The thorax is defined as the carrier body and is attributed to the subindex 0 .

The SC, AC and GH joints are modelled as ideal ball and socket joints and parameterized using the sets of Euler angles.

$$
\theta_{i}=\left(\psi_{i} v_{i} \varphi_{i}\right)^{T}, \quad i=1,2,3 .
$$

The Euler angles are all equal to zero when the corresponding coordinate system is aligned with the initial reference system (attached to the thorax at IJ). The rotation sequences for the SC and AC joints are defined as Y-X-Z. The rotation sequence for the GH joint is defined as Y-X-Y. These angles and sequences are based on the ISB guidelines. Thus, the shoulder's configuration is parameterised by a vector of nine joint angles.

The coordinate transformations between the frames are defined as follows:

$$
\begin{aligned}
& P_{0,1}=\mathbf{R}_{1} P^{1}+P_{1}^{0}, \\
& P_{0,2}=\mathbf{R}_{2} P^{2}+P_{2}^{0}=\mathbf{R}_{2} P^{2}+\mathbf{R}_{1} P_{2}^{1}+P_{1}^{0}, \\
& P_{0,3}=\mathbf{R}_{3} P^{3}+P_{3}^{0}=\mathbf{R}_{3} P^{3}+\mathbf{R}_{2} P_{3}^{2}+\mathbf{R}_{1} P_{2}^{1}+P_{1}^{0}, \\
& \mathbf{R}_{i}=\mathbf{R}\left(\mathrm{z}, \varphi_{i}\right) \mathbf{R}\left(x, v_{i}\right) \mathbf{R}\left(\mathrm{y}, \psi_{i}\right), \\
& \mathbf{R}_{3}=\mathbf{R}\left(\mathrm{y}, \varphi_{3}\right) \mathbf{R}\left(x, v_{3}\right) \mathbf{R}\left(y, \psi_{3}\right),
\end{aligned}
$$

Here $P^{i}$ and $P^{0}{ }_{i}$ represent the vector expressed in the $i^{\text {th }}$ frame $(i=0,1,2,3)$ and the vector from the origin to the $i^{\text {th }}$ frame, respectively. 
The scapulothoracic contact is the contact between the scapula and thorax. The scapula plane can be defined by three bony landmarks TS, AI and AC. The thorax is modelled as an ellipsoid with halfaxis dimensions $a, b$, and $c[19,25,37]$. An additional coordinate frame is attached to the center of the ellipsoid $\mathrm{E}$ with the half-axis dimensions being aligned with the orthogonal axes of the frame. It is known that the intersection of an ellipsoid and a plane is an ellipse (Fig. 2(a)). In the current model, the centre of this small intersection ellipse is considered as the ST contact point. The one ST contact holonomic constraint can be written in the following form:

$$
\Phi_{S T}\left(\theta_{1}, \theta_{2}\right)=\frac{\left(x_{S T}-x_{E}\right)^{2}}{a^{2}}+\frac{\left(y_{S T}-y_{E}\right)^{2}}{b^{2}}+\frac{\left(z_{S T}-z_{E}\right)^{2}}{c^{2}}-1=0,
$$

Here $\left(x_{S T}, y_{S T}, z_{S T}\right)$ and $\left(x_{E}, y_{E}, z_{E}\right)$ are the Cartesian coordinates (vectors $P_{\mathrm{ST}}^{0}$ and $P_{\mathrm{E}}^{0}$ ) of ST point and the centre of ellipsoid $E$, respectively.



Fig. 2. (a) The ellipsoid and plane intersection. (b) The ST point contact in the kinematic shoulder model. (c) The ST contact constraint is replaced by UPS link with passive prismatic joint. Images (b) and (c) were created using OpenSim model.

The forward kinematic analysis of the shoulder model provides a mapping between the nine Euler angles $\left(\theta_{1}, \theta_{2}, \theta_{3}\right)$ at three joints SC, AC and GH and the pose of the end-effecter $P_{\mathrm{HU}}^{0}$ (the HU joint in Fig. 2(b)) subject to one ST contact constraint. Applying the Grubler-Kutzbach criterion [38], the kinematic shoulder model, shown in Fig. 2(b), has 8-DOFs.

$$
F=6(n-j-1)+\sum_{i} f_{i}=6 \cdot(4-4-1)+3 \cdot 3+5=8,
$$

Here $n$ is the number of links including the fixed base, $j$ is the number of joints and $f_{i}$ is their associated DOFs. There are three spherical ball-and-socket joints with 3-DOFs each and there is one constraint, defining the spherical slider joint with 5-DOFs. The self-rotations of the links SC-AC and GH-HU due to spherical joints at both ends introduce two redundant DOFs, called passive DOFs. Hence, the configuration of the end effector (HU joint) is defined by 6-DOFs. The shoulder kinematic model is redundant since the position of $\mathrm{HU}$ joint can be reached with more than one configuration of the shoulder bones. Note that, the axial rotation of the humerus is considered passive as the present work is focussed only on the shoulder movement. The orientation of the humerus must be considered when modelling the elbow (hinge joint).

Further, the ST contact constraint can be replaced with an equivalent kinematic chain [19] comprising of universal joint at the centre of the thorax ellipsoid $E$ connected with the passive prismatic joint to a ball-and-socket joint at the ST point. This additional kinematic chain (ST leg) provides an alternative method for construction of a forward kinematic map. As a result, there are two equivalent kinematic maps: one through the AC joint with ST contact (ellipsoid) constraint and one through ST joint with AC contact (spherical) constraint. For the alternative parallel kinematic map (through ST joint), additional sets of three Euler angles and two spherical angles are defined for the spherical joint at $\mathrm{ST}\left(\psi_{\mathrm{ST}}, v_{\mathrm{ST}}, \varphi_{\mathrm{ST}}\right)$ and for the superimposed universal joint at $\mathrm{E}\left(\alpha_{\mathrm{ST}}, \beta_{\mathrm{ST}}\right)$ 
respectively. To parameterise the final configuration of the humerus (HU joint), two alternative sets of joint coordinates can be used:

$$
\begin{aligned}
& \text { AC map (natural): } q_{A C}=\left(\theta_{1}^{T} \theta_{2}^{T} \theta_{3}^{T}\right)^{T}=\left(\psi_{1} v_{1} \varphi_{1} \psi_{2} v_{2} \varphi_{2} \psi_{3} v_{3} \varphi_{3}\right)^{T} . \\
& \text { ST map (parallel): } q_{S T}=\left(\theta_{1}(1) \alpha_{S T} \beta_{S T} \theta_{S T}^{T} \theta_{3}^{T}\right)^{T}=\left(\varphi_{1} \alpha_{S T} \beta_{S T} \psi_{S T} v_{S T} \varphi_{S T} \psi_{3} v_{3} \varphi_{3}\right)^{T} .
\end{aligned}
$$

Four of the nine joint angles $\left(\varphi_{1}, \psi_{3}, v_{3}, \varphi_{3}\right)$ appear in both Eqs.(8)-(9), the clavicle's axial rotation and three humeral Euler angles, that are unconstrained and mutually independent. The remaining two distinct sets of five joint coordinates correspond to the configuration of the scapula.

Consequently, the shoulder girdle can be represented as a 2-2 parallel mechanism, as shown in Fig. 3 , with the moving platform (scapula) supported by two spherical joints over two legs, one of which is of constant length (clavicle). The scapula is gliding on two surfaces, a sphere and an ellipsoid, through two point contacts: AC and ST. The Grubler-Kutzbach criterion states that the shoulder girdle, without the passive DOF associated with the clavicle's self-rotation, has 4-DOFs (10).

$$
F=6(n-j-1)+\sum_{i} f_{i}=6 \cdot(4-4-1)+3 \cdot 3+1 \cdot 2-1=4 .
$$

For the parallel shoulder girdle, there are two 3-DOFs spherical ball-and-socket joints on the top and one on the base, and there is one 2-DOFs universal base joint superimposed at the ellipsoid's centre E. The prismatic joint in the introduced UPS kinematic chain (ST leg) is not considered as it depends on the universal joint's movement and cannot be actuated separately [19]. The 4-DOFs of the parallel platform correspond to two translational and two rotational DOFs of the scapula, namely: elevation/depression, abduction/adduction, upward/downward rotation and anterior/posterior tilting. The outer link (humerus bone) is serially connected on top of the moving platform through the balland-socket GH joint (as shown in Fig. 3) and its motion is described in terms of three Euler angles $\left(\psi_{3}, v_{3}, \varphi_{3}\right)^{\mathrm{T}}$. Now, the human shoulder can be represented as a hybrid mechanism consisting of a parallel shoulder girdle mechanism which orients and positions the serially connected humerus link.

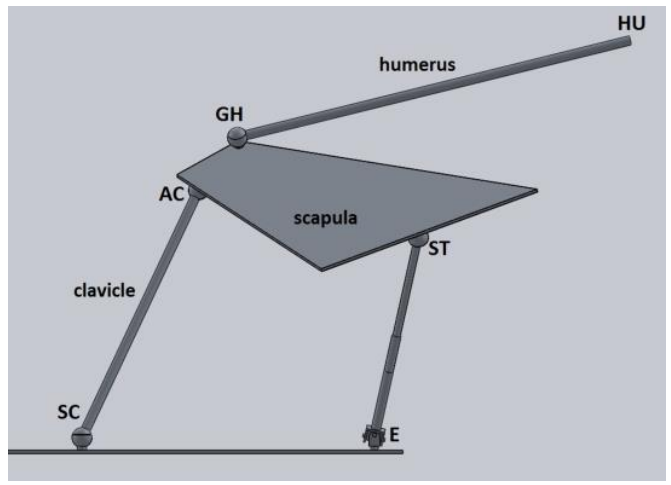

Fig. 3. CAD design of the hybrid shoulder mechanism.

\section{Modelling the human shoulder girdle as a 6-4 parallel mechanism}

\section{Minimal kinematic parameterisation}

In the previous Section, similar to the model in [19], the scapula was parameterised by two alternative sets of five coordinates: $\left(\psi_{1}, v_{1}, \psi_{2}, v_{2}, \varphi_{2}\right)$ and $\left(\alpha_{\mathrm{ST}}, \beta_{\mathrm{ST},} \psi_{\mathrm{ST}}, v_{\mathrm{ST}}, \varphi_{\mathrm{ST}}\right)$. However, as the shoulder girdle has 4-DOFs in the described model, four independent parameters or inputs are needed to fully express the configuration (position and orientation) of scapula. In order to obtain four independent variables to construct a minimal set of parameters, four additional UPS links with active prismatic joints are added to the shoulder girdle parallel platform, as shown in Fig. 4. The new mechanism consists of 12 links: fixed base (thorax), moving platform (scapula), clavicle link, ST leg and 4 additional limbs consisting of two links each (due to the active prismatic joints). There are 16 
joints in total: 6 spherical joints on the moving platform, 5 universal joints and 1 spherical joint on the base, and 4 prismatic joints in the added limbs. In fact, adding an additional UPS link does not alter the total number of DOFs of the spatial manipulator because each UPS link is a complete set of 6DOFs. It can be verified using the mobility formula (11) as follows:

$$
F=6(n-j-1)+\sum_{i} f_{i}=6 \cdot(12-16-1)+7 \cdot 3+5 \cdot 2+4-1=4
$$

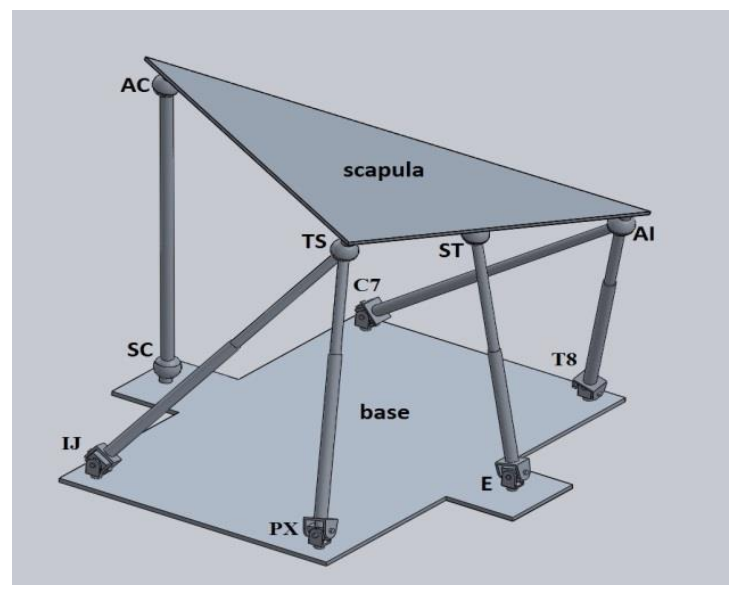

Fig. 4. The human shoulder girdle modelled as a 6-4 parallel mechanism

Thus, the modified parallel mechanism still possesses only 4-DOFs. In contrast, an additional universal-spherical (US) link which has 5-DOFs will reduce the total number of DOFs of the mechanism by one. Note that, as in the previous Section, the passive DOF associated with the clavicle's self-rotation is subtracted and passive prismatic joint in the ST leg is not considered. Moreover, it is also apparent that newly added UPS limbs can be also replaced by SPS limbs without compromising the overall DOFs of the mechanism as the passive DOFs associated with SPS limbs will also be subtracted from the DOF equation. The modified parallel mechanism describing shoulder girdle movement is now modelled as a 6-4 parallel mechanism. Two additional UPS links meet at concentric spherical joints on the mobile platform (scapula) at the bony landmark TS and the other two additional UPS links meet the same way at the scapula landmark AI. The four additional active limbs provide us with four independent inputs in terms of the lengths $d$ of these links. The four links' offsets can be varied independently and are equal to the number of the DOFs of the model.

Due to a special variation of this parallel mechanism, like having constrained links and concentric spherical joints on the top platform, once the lengths of the four additional UPS links are given $\left(d_{1} d_{2} d_{3} d_{4}\right)$, the configuration of the moving platform can be found through the forward kinematics analysis as shown further in this Section.

Consequently, both forward kinematic maps (natural and parallel) described in Section 2 can be parameterised in terms of the following vector of eight minimal coordinates:

$$
q_{\text {min }}=\left(\theta_{1}(1) \mathrm{d}_{1} \mathrm{~d}_{2} \mathrm{~d}_{3} \mathrm{~d}_{4} \theta_{3}{ }^{T}\right)^{T}=\left(\psi_{1} \mathrm{~d}_{1} \mathrm{~d}_{2} \mathrm{~d}_{3} \mathrm{~d}_{4} \psi_{3} v_{3} \varphi_{3}\right)^{T},
$$

\section{Geometry description and kinematics of the 6-4 parallel shoulder girdle mechanism}

The parallel mechanism of the shoulder girdle, shown in Fig. 4, consists of a moving platform (scapula), whose plane is defined using three scapula bony landmarks (AC, TS, and AI), connected to a base platform (thorax) using six limbs: one limb has a constant length and represents the clavicle, one of the limbs with a variable length represents ST contact and modelled as a UPS link with passive prismatic joint and the other four links with variable lengths are modelled as UPS links with active prismatic joints. The four additional universal joints on the base are placed at the defined bony landmarks of the thorax (refer to Fig. 1) to give them real anatomical basis. Thus, the fixed inertial coordinate system (with subindex 0) defined in Section 2 is placed at the base joint of the mechanism (at IJ). Ideally, the four additional base joints (placed at IJ, P8, TX and C7) lie on one plane, called 
sagittal plane, which divides the human body into left and right sides. The initial two base joints, placed at SC joint and at the center of the ellipsoid E, obviously, do not belong to that plane. The model in Fig. 4 is just a simplified representation of the proposed mechanism.

The coordinate frame of the moving platform (with subindex 2) is attached at AC joint as defined in Section 2 (Fig.1). Therefore, the position and orientation of the moving platform (scapula) with respect to the base (thorax) is described by the $4 \times 4$ homogeneous transformation matrix $\mathbf{T}$ which consists of a $3 \times 3$ rotation matrix $\mathbf{R}_{2}$ of the moving platform and a $3 \times 1$ position vector $P_{2}^{0}$ of the AC joint expressed in the inertial frame. The vectors $S C^{0}, P X^{0}, T 8^{0}, C 7^{0}, E^{0}, T S^{2}$ and $A I^{2}$ have constant lengths defined by the dimensions of the shoulder model.

The length of the each limb can be found by taking the dot product of the vector along the limb with itself:

$$
\begin{aligned}
& \mathrm{d}^{2}=\left[P_{2}^{0}-S C^{0}\right]^{T}\left[P_{2}^{0}-S C^{0}\right], \quad \text { for clavicle limb SC-AC } \\
& \mathrm{d}_{1}^{2}=\left[P_{2}^{0}+\mathbf{R}_{2} T S^{2}\right]^{T}\left[P_{2}^{0}+\mathbf{R}_{2} T S^{2}\right], \quad \text { for UPS limb IJ-TS } \\
& \mathrm{d}_{2}^{2}=\left[P_{2}^{0}+\mathbf{R}_{2} T S^{2}-P X^{0}\right]^{T}\left[P_{2}^{0}+\mathbf{R}_{2} T S^{2}-P X^{0}\right], \quad \text { for UPS limb PX-TS } \\
& \mathrm{d}_{3}^{2}=\left[P_{2}^{0}+\mathbf{R}_{2} A I^{2}-C 7^{0}\right]^{T}\left[P_{2}^{0}+\mathbf{R}_{2} A I^{2}-C 7^{0}\right], \quad \text { for UPS limb C7-AI } \\
& \mathrm{d}_{4}^{2}=\left[P_{2}^{0}+\mathbf{R}_{2} A I^{2}-T 8^{0}\right]^{T}\left[P_{2}^{0}+\mathbf{R}_{2} A I^{2}-T 8^{0}\right], \\
& \mathrm{d}_{S T}^{2}=\left[P_{2}^{0}+\mathbf{R}_{2} S T^{2}-E^{0}\right]^{T} \mathbf{E}_{\mathrm{ST}}\left[P_{2}^{0}+\mathbf{R}_{2} S T^{2}-E^{0}\right]-1=0, \\
& \text { Here } \mathbf{E}_{\mathrm{ST}}=\left[\begin{array}{ccc}
\frac{1}{\mathrm{a}^{2}} & 0 & 0 \\
0 & \frac{1}{\mathrm{~b}^{2}} & 0 \\
0 & 0 & \frac{1}{\mathrm{c}^{2}}
\end{array}\right] \quad \text { a, b, c - are ellipsoid half-axis dimensions }
\end{aligned}
$$

The set of the above Eqs (13)-(18) yields six equations describing the pose of the moving platform (scapula) with respect to the fixed base (thorax). It can be seen that the inverse kinematics of such mechanism is simple and gives a unique solution. Given the configuration of the moving platform $\left(\mathbf{R}_{\mathbf{2}}\right.$ and $P_{2}^{0}$ ), the lengths of the links can be found by taking the square root of the above expressions. Thus, there are two possible solutions for the link length. However, only the positive link length is physically feasible and if the solution is a complex number, the configuration of the top platform (scapula) is not reachable.

In contrast to inverse kinematics, forward kinematic (FK) analysis of this kind of parallel mechanism is a challenging task. For a given set of limb lengths, one needs to find the configuration of the moving platform. As stated before, the orientation and position of the moving platform (scapula) can be described using rotation matrix $\mathbf{R}_{2}$ and position vector $P_{2}^{0}$ that contain nine and three scalar unknowns, respectively. There exist different approaches and methods to solve this problem that involves highly nonlinear equations which will lead to multiple solutions.

Nevertheless, in comparison to rotation matrix $\mathbf{R}_{2}$ and position vector $P_{2}^{0}$, the Cartesian coordinates $(\mathrm{x}, \mathrm{y}, \mathrm{z})$ of any three points on the scapula (say AC, TS and AI) expressed in the inertial frame can also fully define the pose of the scapula with respect to the thorax. Then, defining the spatial configuration of all the joints with respect to each other and to the origin (excluding the ST contact point for the moment) will give 8 quadratic equations with 9 unknowns (x, y, z coordinates of 3 moving points). As the location of the ST contact point on the scapula plane is unknown, one ellipsoid constraint equation will add another 3 unknowns ( $\mathrm{x}, \mathrm{y}, \mathrm{z}$ coordinates of the ST contact) resulting in the system of 9 equations with 12 unknowns. In fact, the ST contact point that belongs to the scapula plane can be used to derive additional three equations. As the ST contact belongs to the scapula plane, its coordinates can be expressed in terms of the coordinates of the other three points on the scapula (TS, AI and AC). In other words, the coordinates of the ST contact point are functions of 
the coordinates of the other three scapula points. The vectors that extend from the ST contact point to any of the other three vertices of the scapula triangle are perpendicular to the vector normal to the scapula plane. In other words, the dot products between these vectors and the normal vector must be zero. In turn, the vector normal to the scapula plane can be found using the cross product of any two vectors that connect all three vertices of the scapula triangle as follows:

$$
N^{0}=\left(A I^{0}-A C^{0}\right) \times\left(T S^{0}-A C^{0}\right)
$$

Finally, the system of 12 equations with 12 unknowns (x, y, and $\mathrm{z}$ coordinates of four points) to solve FK is derived as follows (20). Referring to Fig. 4:

$$
\mathbf{F}(\lambda, x)=\left[\begin{array}{c}
\left(\mathrm{X}_{\mathrm{AC}}-\mathrm{X}_{\mathrm{SC}}\right)^{2}+\left(\mathrm{Y}_{\mathrm{AC}}-\mathrm{Y}_{\mathrm{SC}}\right)^{2}+\left(\mathrm{Z}_{\mathrm{AC}}-\mathrm{Z}_{\mathrm{SC}}\right)^{2}-\mathrm{d}^{2} \\
\left(\mathrm{X}_{\mathrm{TS}}\right)^{2}+\left(\mathrm{Y}_{\mathrm{TS}}\right)^{2}+\left(\mathrm{Z}_{\mathrm{TS}}\right)^{2}-\mathrm{d}_{1}^{2} \\
\left(\mathrm{X}_{\mathrm{TS}}-\mathrm{X}_{\mathrm{PX}}\right)^{2}+\left(\mathrm{Y}_{\mathrm{TS}}-\mathrm{Y}_{\mathrm{PX}}\right)^{2}+\left(\mathrm{Z}_{\mathrm{TS}}-\mathrm{Z}_{\mathrm{PX}}\right)^{2}-\mathrm{d}_{2}^{2} \\
\left(\mathrm{X}_{\mathrm{AI}}-\mathrm{X}_{\mathrm{C} 7}\right)^{2}+\left(\mathrm{Y}_{\mathrm{AI}}-\mathrm{Y}_{\mathrm{C} 7}\right)^{2}+\left(\mathrm{Z}_{\mathrm{AI}}-\mathrm{Z}_{\mathrm{C} 7}\right)^{2}-\mathrm{d}_{3}^{2} \\
\left(\mathrm{X}_{\mathrm{AI}}-\mathrm{X}_{\mathrm{T} 8}\right)^{2}+\left(\mathrm{Y}_{\mathrm{AI}}-\mathrm{Y}_{\mathrm{T} 8}\right)^{2}+\left(\mathrm{Z}_{\mathrm{AI}}-\mathrm{Z}_{\mathrm{T} 8}\right)^{2}-\mathrm{d}_{4}^{2} \\
\frac{\left(\mathrm{X}_{\mathrm{ST}}-\mathrm{X}_{\mathrm{E}}\right)^{2}}{\mathrm{a}^{2}}+\frac{\left(\mathrm{Y}_{\mathrm{ST}}-\mathrm{Y}_{\mathrm{E}}\right)^{2}}{\mathrm{~b}^{2}}+\frac{\left(\mathrm{Z}_{\mathrm{ST}}-\mathrm{Z}_{\mathrm{E}}\right)^{2}}{\mathrm{c}^{2}}-1 \\
\left(\mathrm{X}_{\mathrm{TS}}-\mathrm{X}_{\mathrm{AC}}\right)^{2}+\left(\mathrm{Y}_{\mathrm{TS}}-\mathrm{Y}_{\mathrm{AC}}\right)^{2}+\left(\mathrm{Z}_{\mathrm{TS}}-\mathrm{Z}_{\mathrm{AC}}\right)^{2}-\mathrm{L}_{1}^{2} \\
\left(\mathrm{X}_{\mathrm{AI}}-\mathrm{X}_{\mathrm{AC}}\right)^{2}+\left(\mathrm{Y}_{\mathrm{AI}}-\mathrm{Y}_{\mathrm{AC}}\right)^{2}+\left(\mathrm{Z}_{\mathrm{AI}}-\mathrm{Z}_{\mathrm{AC}}\right)^{2}-\mathrm{L}_{2}^{2} \\
\left(\mathrm{X}_{\mathrm{AI}}-\mathrm{X}_{\mathrm{TS}}\right)^{2}+\left(\mathrm{Y}_{\mathrm{AI}}-\mathrm{Y}_{\mathrm{TS}}\right)^{2}+\left(\mathrm{Z}_{\mathrm{AI}}-\mathrm{Z}_{\mathrm{TS}}\right)^{2}-\mathrm{L}_{3}^{2} \\
\mathrm{X}_{\mathrm{N}} \cdot\left(\mathrm{X}_{\mathrm{ST}}-\mathrm{X}_{\mathrm{AC}}\right)+\mathrm{Y}_{\mathrm{N}} \cdot\left(\mathrm{Y}_{\mathrm{ST}}-\mathrm{Y}_{\mathrm{AC}}\right)+\mathrm{Z}_{\mathrm{N}} \cdot\left(\mathrm{Z}_{\mathrm{ST}}-\mathrm{Z}_{\mathrm{AC}}\right) \\
\mathrm{X}_{\mathrm{N}} \cdot\left(\mathrm{X}_{\mathrm{ST}}-\mathrm{X}_{\mathrm{AI}}\right)+\mathrm{Y}_{\mathrm{N}} \cdot\left(\mathrm{Y}_{\mathrm{ST}}-\mathrm{Y}_{\mathrm{AI}}\right)+\mathrm{Z}_{\mathrm{N}} \cdot\left(\mathrm{Z}_{\mathrm{ST}}-\mathrm{Z}_{\mathrm{AI}}\right) \\
\mathrm{X}_{\mathrm{N}} \cdot\left(\mathrm{X}_{\mathrm{ST}}-\mathrm{X}_{\mathrm{TS}}\right)+\mathrm{Y}_{\mathrm{N}} \cdot\left(\mathrm{Y}_{\mathrm{ST}}-\mathrm{Y}_{\mathrm{TS}}\right)+\mathrm{Z}_{\mathrm{N}} \cdot\left(\mathrm{Z}_{\mathrm{ST}}-\mathrm{Z}_{\mathrm{TS}}\right)
\end{array}\right]=\mathbf{0}
$$

The system of Eq. (20) is a set of fundamental equations that has 12 polynomial equations with a highest order of 2, where $\lambda=\left[X_{A C} Y_{A C} Z_{A C} X_{T S} Y_{T S} Z_{T S} X_{A I} Y_{A I} Z_{A I} X_{S T} Y_{S T} Z_{S T}\right]^{T}$ is the 12-dimensional output vector of the unknowns and $x=\left[\mathrm{d}_{1} \mathrm{~d}_{2} \mathrm{~d}_{3} \mathrm{~d}_{4}\right]^{T}$ is the four-dimensional input vector of the link lengths variables. Other symbols in Eq. (20) represent constant values. Note that, if the base points IJ, P8, TX and C7 lie on one plane, their z-components become zero. The representation of Eq. (20) in a matrix form is adopted from [39]. This system of equations incorporates ellipsoid constraint and allows the ST contact to move on the scapula plane. Thus, the input values $x=\left[\mathrm{d}_{1} \mathrm{~d}_{2} \mathrm{~d}_{3} \mathrm{~d}_{4}\right]^{T}$ that solve Eq.(20) can be referred as the minimal coordinates that incorporate the ST constraint. Such systems of highly nonlinear equations can be solved using different analytical methods [37-40]. This can result in a number of possible forward kinematic solutions. However, a numerical iteration method with the appropriate initial guess vector can be applied to find the current forward kinematic solution of the moving platform that lies in the same branch of solutions as the initial configuration of the parallel platform [40]. Note that, if the location of the ST contact point is predefined and fixed on the scapula plane, the system of equations will be simplified to a system of 9 equations with 9 unknowns. A derivation of the closed-form expression is considerably simplified for this case.

Once the Cartesian coordinates (in inertial frame) of three points (AC, TS and AI) on the scapula are found, the orientation matrix $\mathbf{R}_{\mathbf{2}}$ and position vector $P_{2}^{0}$ can be derived as follows:

- The unit vector along the vector TS-AC is the 3rd column of matrix $\mathbf{R}_{\mathbf{2}}$

$$
\mathbf{R}_{2}(:, 3)=\left(\frac{\mathrm{X}_{\mathrm{AC}}-\mathrm{X}_{\mathrm{TS}}}{\mathrm{L}_{1}}, \frac{\mathrm{Y}_{\mathrm{AC}}-\mathrm{Y}_{\mathrm{TS}}}{\mathrm{L}_{1}}, \frac{\mathrm{Z}_{\mathrm{AC}}-\mathrm{Z}_{\mathrm{TS}}}{\mathrm{L}_{1}}\right)
$$

- The unit vector along the cross product of vectors TS-AC $(u)$ and TS-AI $(w)$ is the 1 st column of matrix $\mathbf{R}_{\mathbf{2}}$

$$
\mathbf{R}_{\mathbf{2}}(:, 1)=\left(\frac{\left(u_{y} w_{z}-u_{z} w_{y}\right)}{|(u \times w)|}, \frac{\left(u_{z} w_{x}-u_{x} w_{z}\right)}{|(u \times w)|}, \frac{\left(u_{x} w_{y}-u_{y} w_{x}\right)}{|u \times w|}\right)
$$


- The 3rd unit vector that can be found as the cross product of the above two is the 2 nd column of matrix $\mathbf{R}_{\mathbf{2}}$.

$$
\mathbf{R}_{\mathbf{2}}(:, 2)=\mathbf{R}_{\mathbf{2}}(:, 3) \times \mathbf{R}_{\mathbf{2}}(:, 1)
$$

The position vector $P_{2}^{0}=\left(\mathrm{X}_{\mathrm{AC}}, \mathrm{Y}_{\mathrm{AC}}, \mathrm{Z}_{\mathrm{AC}}\right)$ is derived from the Cartesian coordinates of the AC joint. It can be seen that, in all cases, the input set of link lengths $\left(\mathrm{d}_{1}, \mathrm{~d}_{2}, \mathrm{~d}_{3}, \mathrm{~d}_{4}\right)$ is directly related to the transformation matrix (rotation matrix $\mathbf{R}_{\mathbf{2}}$ and position vector $P_{2}^{0}$ ) which, in turn, contains all the shoulder girdle joint angles, described in Section 2.

Once the configuration of the moving platform is known, any point, for instance GH joint, defined in scapula's coordinate frame $B$ can be found as follows:

$$
G H^{0}=P_{2}^{0}+\mathbf{R}_{2} G H^{2}
$$

\section{Shoulder motion planning with the proposed shoulder girdle mechanism}

As stated in Section 2, the kinematic model of the human shoulder is redundant: there exist multiple sets of joint angles for a given pose of the upper arm. The motion planning in musculoskeletal shoulder models is a challenging task due to the presence of the ST constraint(s). When constraints are not considered, the kinematic model's nine joint angles $(q)$ are independent and can be ascribed the values of the measured angles $\left(q_{m}\right)$. When constraints are considered, the joint coordinates become interdependent and the motion planning requires data-driven optimisation to minimise the error between the model's coordinates $q$ and the measured values of the coordinates $q_{m}$ at discrete instances of the motion [22, 29, 34, 41-43]. Thus, this kind of approach requires the availability of the measured data to solve the optimisation problem at every instant of the movement. The minimal coordinates presented in this work are independent from each other and, when used to solve Eq. 20, incorporate the constraints. Hence, if measured movement is expressed in terms of the proposed minimal coordinates it can be directly imposed on the model.

In this Section the proposed model is used for humeral abduction (from $0^{\circ}$ to $160^{\circ}$ ) in the scapular plane. The method of planning the model's kinematics is adapted from [41] but it is now applied to the proposed shoulder model with one ST contact constraint utilizing a novel set of minimal coordinates presented in the previous Section. The independent variables $d_{1}, d_{2}, d_{3}$ and $d_{4}$ parameterise shoulder girdle motion and are equivalent to the five joint angles $\left(\psi_{1} v_{1} \psi_{2} v_{2} \varphi_{2}\right)$. First, the motion is planned in terms of the minimal coordinates $q_{\min }$ and constructed in terms of $q_{\min }(t)$ which is then mapped back to $q(t)$. Using the shoulder girdle minimal coordinates, the spatial locations of the scapula points can be found and the joint angles can be extracted from the rotation matrices $\mathbf{R}_{\mathbf{1}}$ and $\mathbf{R}_{\mathbf{2}}$ knowing their sequence of rotation.

The time-dependent parameterisation of the minimal coordinates is defined using the dataset from the literature [22] that contains positions of all the required anatomical landmarks and the dimensions of the ellipsoid. The minimal set of coordinates $q_{\min }(t)$ is planned corresponding to the description of humeral abduction in [41]. From $30^{\circ}$ to $160^{\circ}$ abduction, the shoulder girdle's parameters are planned as a linear function of time. The clavicle's axial rotation coordinate $\varphi_{1}$ is held constant during the first $30^{\circ}$ humeral abduction and then rotated posteriorly by $40^{\circ}$ using a linear function of time. In order to simulate the arm abduction in the scapular plane the glenohumeral joint angles $\psi_{3}$ and $\varphi_{3}$ are held constant at $0^{\circ}$ and $30^{\circ}$ whereas the third glenohumeral angle $v_{3}$ is planned using a linear function of time.

$$
\varphi_{1}(t)= \begin{cases}0^{\circ} & t \in\left[0, \frac{30}{160}\right] \\ 0^{\circ}+40^{\circ} t, & t \in\left[\frac{30}{160}, 1\right]\end{cases}
$$




$$
\begin{aligned}
& \mathrm{d}_{i}(t)=\left\{\begin{array}{ll}
\mathrm{d}_{i}(0) & t \in\left[0, \frac{30}{160}\right] \\
\mathrm{d}_{i}(0)+\left(\mathrm{d}_{i}(1)-\mathrm{d}_{i}(0)\right) t, & t \in\left[\frac{30}{160}, 1\right]
\end{array} \quad i=1-4 .\right. \\
& \psi_{3}(t)=0^{\circ}, \quad v_{3}(t)=0^{\circ}+160^{\circ} t, \quad \varphi_{3}(t)=30^{\circ}, \quad t \in[0,1]
\end{aligned}
$$

As the minimal coordinates presented in this work are defined as the distances between the main anatomical landmarks, the initial values $\mathrm{d}_{1}(0), \mathrm{d}_{2}(0), \mathrm{d}_{3}(0)$ and $\mathrm{d}_{4}(0)$ are obtained from the existing dataset of the bony landmarks (Table 2 in [22]). The values $d_{1}(1), d_{2}(1), d_{3}(1)$ and $d_{4}(1)$ are set according to the measured final pose of the shoulder at $160^{\circ}$ humeral abduction. The values $\mathrm{d}_{1}(t)$, $\mathrm{d}_{2}(t), \mathrm{d}_{3}(t)$ and $\mathrm{d}_{4}(t)$ are calculated using Eqs.(26)-(29) for $t=0^{\circ}, 45^{\circ}, 90^{\circ}, 120^{\circ}, 140^{\circ}$ and $160^{\circ}$. These sets of four minimal coordinates $\left(\mathrm{d}_{1}, \mathrm{~d}_{2}, \mathrm{~d}_{3}, \mathrm{~d}_{4}\right)$ are used as inputs in Eq. (20) to obtain the spatial locations of the scapula landmarks AC, TS, AI and ST at each instant of the movement. Eq. (20) is solved in MATLAB using fsolve function with the initial guess vector (initial coordinates of the scapula landmarks). The initial estimate of the ST contact is chosen to be the centroid of the scapula plane. Once the geometric location of the three scapula points AC, TS and AI is obtained, the rotation matrix $\mathbf{R}_{2}$ is constructed using Eqs. (21)-(23). To be consistent with the ISB recommendations [35] where the scapula reference frame is defined using the scapula landmark AA, the spatial coordinate of this point is found using Eq. (24). The new rotation matrix $\mathbf{R}_{2}{ }^{*}$ is then constructed from the scapula points AC, TS and AA. Finally, the scapula joint angles are extracted from the rotation matrix using inverse trigonometric functions knowing that the sequence is $\mathrm{Y}-\mathrm{X}-\mathrm{Z}$, as stated earlier in Section 2. The obtained scapular angles during the humeral abduction are shown in Figs. 5.



(a)

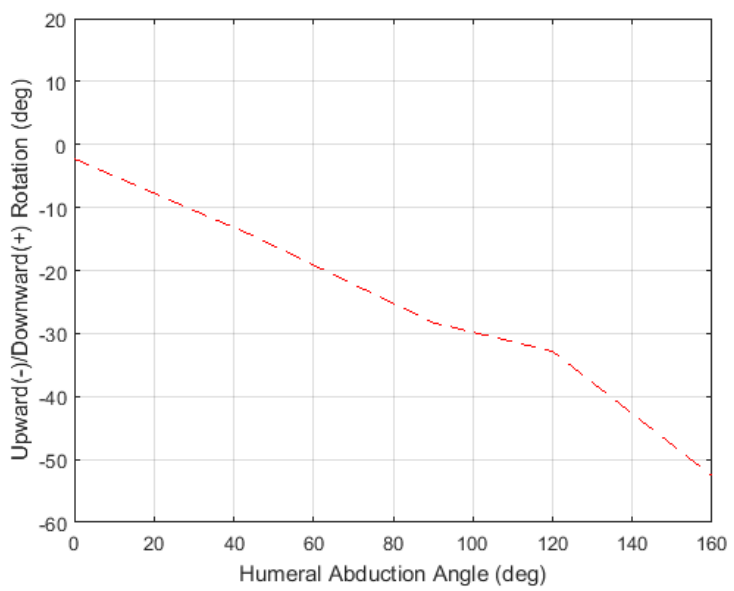

(b) 


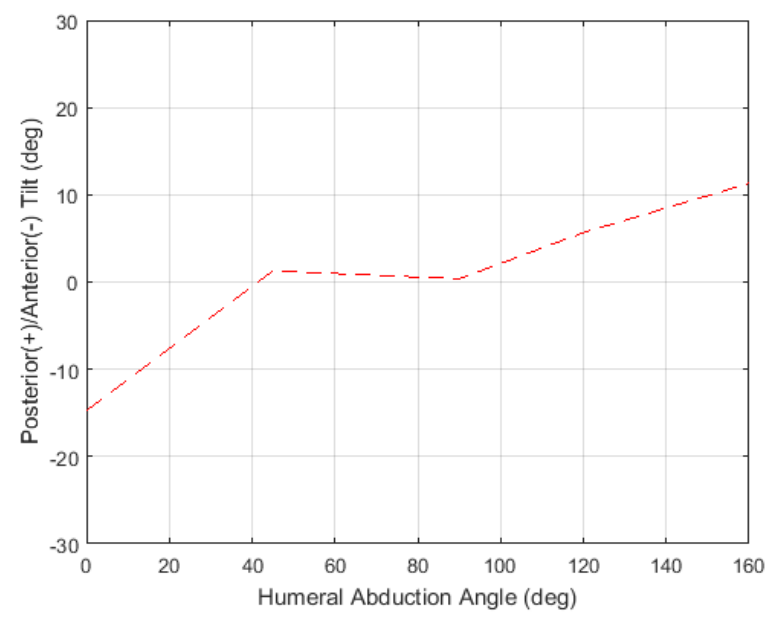

(c)

Fig. 5. Scapular joint angles during humeral abduction. (a) Scapular internal rotation, $v_{2}$. (b) Scapular upward rotation, $\psi_{2}$. (c) Scapular posterior tilting, $\varphi_{2}$.

At $0^{\circ}$ humeral abduction (initial position), the scapula is rotated internally $31^{\circ}$, upwardly $2^{\circ}$ and tilted anteriorly $14^{\circ}$. At $160^{\circ}$ humeral abduction (final position), the scapula is rotated internally $57^{\circ}$, upwardly $52^{\circ}$ and tilted posteriorly $11^{\circ}$. Hence, the scapula is internally and upwardly rotated and posteriorly tilted during humeral abduction. The largest change $\left(50^{\circ}\right)$ between the initial and final values is shown by the upward rotation angle (Fig. 5(b)). Despite the recommendations set by ISB for the shoulder, the direct comparison between the existing studies in the literature is problematic due to the methodologic differences: definition of the initial position, orientation of the coordinate systems, Euler angle sequences, geometrical parameters, variability in marker placement, etc. In this study, the choice of the initial guess vector can also affect the numerical calculations. Nevertheless, the general course of the computed scapula joint angles in the presented work is in agreement with the literature $[22,44-46]$.

The movement of the ST contact point during humeral abduction on a scapula plane (defined by points AC, TS and AI) is shown with arrow in Fig. 6. The ST contact point has been close to the centre of the scapula plane and moved only $16.65 \mathrm{~mm}$ during full humeral abduction. This seems to support the study in [26] where it has been found that the centre of the inner scapular plane had small deviations in distance and angle with respect to the thorax.

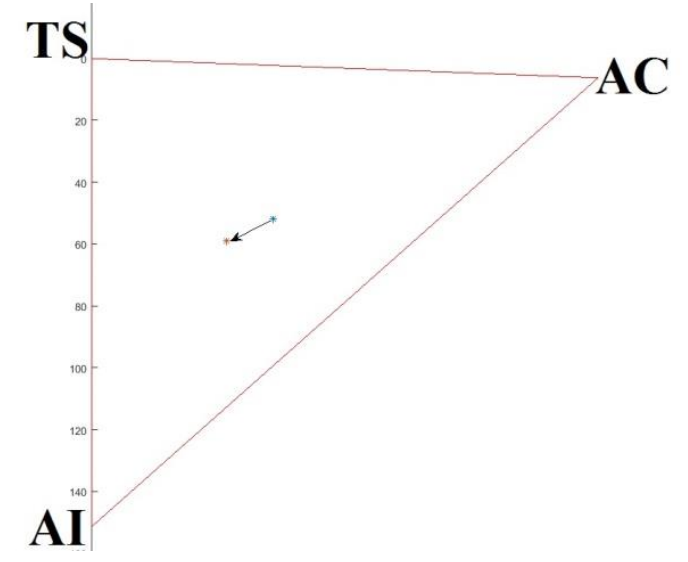

Fig. 6. The movement of the ST contact during humeral abduction (from $0^{\circ}$ to $160^{\circ}$ ) on a scapula plane. 


\section{Discussion}

As stated earlier, the contact between the scapula bone and thorax, which is not a joint in anatomical sense, complicates the shoulder kinematics and introduces the constraints to the existing shoulder biomechanical models. In fact, the shoulder models become less reliable when the scapulothoracic constraints are not considered [29]. To simplify the shoulder motion planning and remove the interdependencies between the joint coordinates, minimal parameterisation in terms of independent variables, which incorporate the model constraints and are equal to the number of DOFs, is needed. The advantages of using the minimal coordinates for the shoulder motion planning are introduced in [19] where three sets of seven minimal coordinates (three of which are for the shoulder girdle) were derived for a 7-DOFs shoulder model with two ST contact constraints. In order to construct the dynamic model, one needs to map the minimal coordinates back to the joint angle parameterisation as the kinematic chains defined to model the ST contact points would cause the physical inconsistencies such as a tensile force in the scapula whereas there is only a compressive one.

The single ST contact point model used to describe the scapulothoracic articulation in this paper led to the kinematic shoulder model with 8-DOFs, four of which correspond to the shoulder girdle. After replacing the single ST contact constraint with the equivalent kinematic chain the pose of the humerus was parameterised using two alternative forward kinematic maps. It was then shown that, adding redundant UPS links with active prismatic joints to the 2-2 parallel mechanism does not alter the number of DOFs of the mechanism. Moreover, the variable link lengths of these additional kinematic chains that are independent of each other and are equal to the number of DOFs can serve as the minimal set of input parameters for the shoulder girdle parallel mechanism. Thus, in contrast to the three sets of minimal coordinates proposed in [19], a single common set of minimal coordinates for both forward kinematic maps was presented in this paper. Indeed, the idea of adding redundant parallel kinematic links to the closed kinematic chains can be generalised and the approach presented in this work could form the basis of a general methodology of formulating parameterisations of kinematic models with closed kinematic chains.

Consequently, the human shoulder girdle is modelled as a 6-4 parallel mechanism. In fact, the geometry of the additional attachment points on the base could be adjusted in different ways, e.g. in case when two base joints are placed at one concentric joint on the base the structure of the parallel mechanism will be described as 5-4. However, the shifts in joint locations will not change the kinematic properties of the mechanism. The choice of the real bony landmarks for the base joints is made to facilitate the application of the model in the studies of the shoulder kinematics. The detailed kinematic analysis of the proposed parallel mechanism is carried out to provide more insight on kinematic characteristics of the human shoulder. It might be claimed that the feasible mechanical system can be constructed with similar kinematic characteristics to those of the human shoulder girdle. The equations of forward kinematics present a novel approach to estimate the spatial configuration of the scapula allowing the gliding motion of the ST contact point while respecting the surface constraint. In addition, the proposed shoulder girdle mechanism can be used to track the ST contact motion during a given shoulder movement which opens a new prospect in shoulder biomechanics. Also, a moving ST contact point could improve the prediction of muscular moment arms providing more anatomically real musculoskeletal models [29]. Another advantage of the proposed parallel mechanism is that it could be adapted to examine pathological cases, e.g. "winging" scapula, by locating the ST contact point further away from the medial border. In that case, the medial border of the scapula will be able to lift off the thorax surface.

The case study on shoulder motion planning shows that the proposed model can be used to predict shoulder kinematics during a given movement. The existing methods to predict shoulder motions use minimisation with respect to measured kinematics or regression models which do not consider the kinematic constraints. The advantage of the proposed model is that it directly incorporates the moving ST contact constraint and simplifies the motion planning without the need of the measured data at every instant of time. In this regard, the proposed method of shoulder planning using the independent minimal coordinates can be used to correct the limitations of the regression models and this opens another appealing perspective. Moreover, the presented minimal coordinates parameterise the movement of bony landmarks and are apparently applicative for skin marker palpation techniques. The simplicity with which they can be applied also makes them attractive. The future work can 
include the workspace, singularity, Jacobian and dynamic analyses of the proposed parallel mechanism. A closed-form solution of Eq. (20) can give more insight on the kinematic properties of the shoulder girdle mechanism. The forces in the actuated limbs in the shoulder girdle parallel mechanism can be regarded as resultant forces from the shoulder muscles acting on the scapula as the number of the shoulder muscles involved in the upper limb motions is much greater than the shoulder DOFs. Finally, the humanoid robots can also replicate this feasible parallel architecture to build the robotic shoulder girdle mechanism.

\section{Conclusions}

The human shoulder girdle can be considered as a closed kinematic chain considering the contact between the scapula and thorax and modelled as a parallel mechanism. The kinematic model of the human shoulder in this paper is based on the model with one point scapulothoracic contact constraint which makes the shoulder girdle a 4-DOFs parallel mechanism. It is shown that, by imposing additional kinematic chains that do not change the number of DOFs, the shoulder girdle can be modelled as a 6-4 parallel mechanism. Moreover, the redundant link lengths can provide the minimal set of independent coordinates and can be used to facilitate the shoulder motion planning while abiding by the moving scapulothoracic joint constraint.

\section{Acknowledgements}

This research is supported by the seed funding jointly provided by Faculty of Engineering and Information Sciences, and School of Mechanical, Materials, Mechatronic and Biomedical Engineering, and the PhD scholarship for the first author funded by the School of Mechanical, Materials, Mechatronic and Biomedical Engineering, University of Wollongong.

\section{References}

[1] B. Bolsterlee, D. H. Veeger, and E. K. Chadwick, "Clinical applications of musculoskeletal modelling for the shoulder and upper limb," Med Biol Eng Comput, vol. 51, pp. 953-63, Sep 2013.

[2] C. Hogfors, G. Sigholm, and P. Herrerts, "Biomechanical model of the human shoulder - i. Elements," J Biomech, vol. 20, pp. 157-166, 1987.

[3] C. Hogfors, B. Peterson, G. Sigholm, and P. Herberts, "Biomechanical model of the human shoulder joint - II. The shoulder rhythm," J Biomech, vol. 24, pp. 699-709, 1991.

[4] A. A. Nikooyan, H. E. Veeger, E. K. Chadwick, M. Praagman, and F. C. Helm, "Development of a comprehensive musculoskeletal model of the shoulder and elbow," Med Biol Eng Comput, vol. 49, pp. 1425-35, Dec 2011.

[5] F. C. Van der Helm, "Analysis Of The Kinematic And Dynamic Behavior Of The Shoulder Mechanism," J Biomech, vol. 27, pp. 527-550, 1994.

[6] G. R. Johnson and I. W. Charlton, "A model for the prediction of the forces at the glenohumeral joint," Proceedings of the Institution of Mechanical Engineers, Part H: Journal of Engineering in Medicine, vol. 220, pp. 801-812, 2006.

[7] K. R. S. Holzbaur, W. M. Murray, and S. L. Delp, "A Model of the Upper Extremity for Simulating Musculoskeletal Surgery and Analyzing Neuromuscular Control," Annals of Biomedical Engineering, vol. 33, pp. 829-840, 2005.

[8] M. Damsgaard, J. Rasmussen, S. T. Christensen, E. Surma, and M. de Zee, "Analysis of musculoskeletal systems in the AnyBody Modeling System," Simulation Modelling Practice and Theory, vol. 14, pp. 1100-1111, 2006.

[9] B. A. Garner and M. G. Pandy, "Musculoskeletal model of the upper limb based on the visible human male dataset," Comput Methods Biomech Biomed Engin, vol. 4, pp. 93-126, Feb 2001.

[10] C. R. Dickerson, D. B. Chaffin, and R. E. Hughes, "A mathematical musculoskeletal shoulder model for proactive ergonomic analysis," Comput Methods Biomech Biomed Engin, vol. 10, pp. 389-400, Dec 2007.

[11] J. Lenarcic and A. Umek, "Simple Model of Human Arm Reachable Workspace," IEEE TRANSACTIONS ON SYSTEMS, MAN \& CYBERNETICS, vol. 24, 1994.

[12] J. Yang, K. Abdel-Malek, and K. Nebel, "Reach envelope of a 9-degree-of-freedom model of the upper extremity," International Journal of Robotics and Automation, vol. 20, 2005.

[13] N. Klopcar, M. Tomsic, and J. Lenarcic, "A kinematic model of the shoulder complex to evaluate the arm-reachable workspace," J Biomech, vol. 40, pp. 86-91, 2007.

[14] J. Lenarcic and M. Stanisic, "A humanoid shoulder complex and the humeral pointing kinematics," IEEE Trans Robot Autom, vol. 19, 2003.

[15] A. E. Engin and S. T. Tumer, "Three-dimensional kinematic modelling of the human shoulder complex - part I: physical model and determination of joint sinus cones," Journal of Biomechanical Engineering, vol. 111, 1989.

[16] B. Tondu, "Modelling of the Shoulder Complex and Application to the Design of Upper Extremities for Humanoid Robots," in 5th IEEE-RAS Int. Conf. on Human Robots, 2005.

[17] E. Berthonnaud, G. Herzberg, K. D. Zhao, K. N. An, and J. Dimnet, "Three-dimensional in vivo displacements of the shoulder complex from biplanar radiography," Surg Radiol Anat, vol. 27, pp. 214-22, Aug 2005. 
[18] J. Lenarcic, M. Stanisic, and V. Parenti-Castelli, "Kinematic design of a humanoid robotic shoulder complex," in ICRA, San Francisco, CA, USA, 2000.

[19] D. Ingram, C. Engelhardt, A. Farron, A. Terrier, and P. Müllhaupt, "Modelling of the human shoulder as a parallel mechanism without constraints," Mechanism and Machine Theory, vol. 100, pp. 120-137, 2016.

[20] W. Maurel and D. Thalmann, "Human shoulder modeling including scapulo-thoracic constraint and joint sinus cones," Computers \& Graphics, vol. 24, pp. 203-218, 2000.

[21] Y. Kitano and K. Yokota, "Method for Measuring Position and Posture of the Shoulder Skeleton Using Parallel Links," The Japan Society of Mechanical Engineers, 2012.

[22] A. El Habachi, S. Duprey, L. Cheze, and R. Dumas, "A parallel mechanism of the shoulder - application to multi-body optimisation," Multibody System Dynamics, vol. 33, pp. 439-451, 2014.

[23] C. Charbonnier, S. Chague, F. C. Kolo, J. C. Chow, and A. Ladermann, "A patient-specific measurement technique to model shoulder joint kinematics," Orthop Traumatol Surg Res, vol. 100, pp. 715-9, Nov 2014.

[24] W. Maurel, "3D modeling of the human upper limb including the biomechanics of joints, muscles and soft tissues," Doctor of Science, 1998.

[25] B. A. Garner and M. G. Pandy, "A Kinematic Model of the Upper Limb Based on the Visible Human Project (VHP) Image Dataset," Comput Methods Biomech Biomed Engin, vol. 2, pp. 107-124, 1999.

[26] S. Sah and X. Wang, "Determination of geometric constraints between the ribcage and scapula in the shoulder complex: a cadaver study," Computer Methods in Biomechanics and Biomedical Engineering, vol. 12, pp. 223-224, 2009.

[27] D. Blana, J. G. Hincapie, E. K. Chadwick, and R. F. Kirsch, "A musculoskeletal model of the upper extremity for use in the development of neuroprosthetic systems," J Biomech, vol. 41, pp. 1714-21, 2008.

[28] A. Seth, R. Matias, A. P. Veloso, and S. L. Delp, "A Biomechanical Model of the Scapulothoracic Joint to Accurately Capture Scapular Kinematics during Shoulder Movements," PLoS One, vol. 11, p. e0141028, 2016.

[29] A. Naaim, F. Moissenet, S. Duprey, M. Begon, and L. Cheze, "Effect of various upper limb multibody models on soft tissue artefact correction: A case study," J Biomech, Jan 312017.

[30] J. H. de Groot, "A Three-Dimensional regression model of the scapulo-humeral rhythm," in 1st Conf. of the ISG.

[31] X. Xu, J. H. Lin, and R. W. McGorry, "A regression-based 3-D shoulder rhythm," J Biomech, vol. 47, pp. 1206-10, Mar 212014.

[32] K. R. Saul, X. Hu, C. M. Goehler, M. E. Vidt, M. Daly, A. Velisar, et al., "Benchmarking of dynamic simulation predictions in two software platforms using an upper limb musculoskeletal model," Comput Methods Biomech Biomed Engin, vol. 18, pp. $1445-58,2015$.

[33] D. Karlsson and B. Peterson, "Towards a model for force predictions in the human shoulder," J Biomech, vol. 25, pp. 189-199, 1992.

[34] V. Sholukha, B. Bonnechere, P. Salvia, F. Moiseev, M. Rooze, and S. Van Sint Jan, "Model-based approach for human kinematics reconstruction from markerless and marker-based motion analysis systems," J Biomech, vol. 46, pp. 2363-71, Sep 27 2013.

[35] G. Wu, F. C. T. van der Helm, H. E. J. Veeger, M. Makhsous, P. Van Roy, C. Anglin, et al., "ISB recommendation on definitions of joint coordinate systems of various joints for the reporting of human joint motion-Part II: shoulder, elbow, wrist and hand," $J$ Biomech, vol. 38, pp. 981-992, 2005.

[36] S. L. Delp, F. C. Anderson, A. S. Arnold, P. Loan, A. Habib, C. T. John, et al., "OpenSim Open Source Software to Create and analyze dynamic simulations of movement," IEEE Trans Biomed Eng, vol. 54, 2007.

[37] F. C. van der Helm, "A FINITE ELEMENT MUSCULOSKELETAL MODEL OF THE SHOULDER MECHANISM," $J$ Biomech, vol. 27, 1994.

[38] L.-W. Tsai, Robot Analysis: The Mechanics of Serial and Parallel Manipulators, 1999.

[39] L. Gao and W. Wu, "Forward kinematics modeling of spatial parallel linkage mechanisms based on constraint equations and the numerical solving method," Robotica, vol. 35, pp. 293-309, 2015.

[40] A. Niyetkaliyev and A. Shintemirov, "An Approach for Obtaining Unique Kinematic Solutions of a Spherical Parallel Manipulator," in Proc. IEEE/ASME Int. Conf. Adv. Int. Mechatronics, Besancon, France, 2014, pp. 1355-1360.

[41] D. Ingram, "Musculoskeletal model of the human shoulder for joint force estimation," PhD, 2015.

[42] B. Bolsterlee, H. E. Veeger, and F. C. van der Helm, "Modelling clavicular and scapular kinematics: from measurement to simulation," Med Biol Eng Comput, vol. 52, pp. 283-91, Mar 2014.

[43] A. Cereatti, T. Bonci, M. Akbarshahi, K. Aminian, A. Barre, M. Begon, et al., "Standardization proposal of soft tissue artefact description for data sharing in human motion measurements," J Biomech, Feb 212017.

[44] P. W. McClure, L. A. Michener, B. J. Sennett, and A. R. Karduna, "Direct 3-dimensional measurement of scapular kinematics during dynamic movements in vivo," J Shoulder Elbow Surg, vol. 10, pp. 269-77, May-Jun 2001.

[45] A. R. Karduna, P. W. McClure, and L. Michener, "Scapular kinematics: e!ects of altering the Euler angle sequence of rotations," J Biomech, vol. 33, pp. 1063-1068, 2000.

[46] K. F. MacLean, J. N. Chopp, T. J. Grewal, B. R. Picco, and C. R. Dickerson, "Three-dimensional comparison of static and dynamic scapular motion tracking techniques," J Electromyogr Kinesiol, vol. 24, pp. 65-71, Feb 2014. 